\title{
Autophagy: Vanquisher of the "Unseen Dangers" in Neurodegenerative Diseases?
}

\author{
Koon AC* \\ School of Life Sciences, The Chinese University of Hong Kong, Hong Kong \\ *Corresponding author: Alex Chun Koon, PhD, School of Life Sciences, The Chinese \\ University of Hong Kong, Hong Kong, Email: alexkoon@cuhk.edu.hk
}

Mini Review

Volume 2 Issue 3

Received Date: May 14, 2019

Published Date: May 22, 2019

\section{Abstract}

Aggregation of misfolded proteins is a hallmark of many neurodegenerative diseases, such as Alzheimer's disease, Parkinson's disease, amyotrophic lateral sclerosis and polyglutamine (polyQ) diseases. Clearance of misfolded proteins in the cell relies on the ubiquitin-proteasome system (UPS) and the autophagy-lysosome system. Since their discoveries, the UPS and autophagy were once thought to be independent of each other in terms of components, mechanisms and substrate selectivity. The UPS was believed to be responsible for degrading soluble proteins, whereas autophagy for degrading insoluble protein aggregates. However, recent studies have demonstrated that the insoluble protein aggregates may not be cytotoxic. By contrast, after deaggregation, multimers and microaggregates may still be present in the cell causing cytotoxicity. The UPS is incapable of degrading these soluble and semi-soluble species, and the vital task of degradation these toxic species rests upon the autophagy-lysosome system. Therefore, apart from degrading the visible protein aggregates, autophagy is also responsible for eliminating these "invisible dangers" to protect the cell in neurodegenerative diseases.

Keywords: Autophagy; Protein Aggregation; Polyglutamine; Neurodegeneration

\section{Introduction}

Aggregation of misfolded proteins is a common pathological feature shared by many neurodegenerative diseases, including but not limited to Alzheimer's disease, Parkinson's disease, amyotrophic lateral sclerosis and polyglutamine (polyQ) diseases [1]. The prolonged accumulation of protein aggregates leads to endoplasmic reticulum stress, neuronal dysfunction, neurodegeneration and eventually cell death [2]. To degrade harmful misfolded proteins, the cell utilizes two major intracellular quality control and recycling systems
- the ubiquitin-proteasome system (UPS) and the autophagy-lysosome system [3]. The UPS is a selective proteolytic system in which utilizes ubiquitination to tagged specific targets for degradation by the proteasome $[4,5]$. Autophagy also utilizes ubiquitination to label targets, but it is a bulk degradative system that uses lysosomal hydrolases to degrade proteins as well as various other cellular constituents [5]. Since their discoveries, the UPS and autophagy were once thought to be independent of each other in terms of components, mechanisms and substrate selectivity. The UPS relies on proteasomes to degrade proteins. Its substrates must be 
small enough to fit into the degradative channel of the proteasomes. Therefore, it is believed that the UPS is mostly responsible for degrading soluble proteins. By contrast, autophagy has the ability to degrade large aggregates via autophagosomes. Thus autophagy was originally viewed as the means to degrade insoluble protein aggregates. However, recent studies indicate that autophagy may participate in the clearance of toxic soluble protein multimers and semi-soluble microaggregates that are often under the radar due to difficulty of detection using traditional laboratory techniques (e.g. immunocytochemistry and filter trap assay). In this review, we highlight some of the recent research advances that prompted us to revisit our understanding of the two protein degradation systems.

\section{Misfolded Proteins are Degraded by the UPS and Autophagy}

In cells, the two major molecular systems responsible for the clearance of misfolded proteins are: (1) the ubiquitin-proteasome system (UPS) and (2) the autophagy-lysosome system (a.k.a. autophagy) [3]. In brief, in the UPS, ubiquitins covalently attach onto an unwanted/misfolded protein substrate to label it for degradation. The ubiquitinated substrate is then delivered to the $26 \mathrm{~S}$ proteasome. The substrate then passes through the degradative channel of the proteasome, and the protein is broken down into amino acids, ready to be recycled $[4,5]$. Comparing to autophagy, the UPS is usually the preferred clearance route of unwanted proteins since it is much more rapid in protein degradation. However, if the misfolded proteins form insoluble aggregates, then they may not be able to enter the degradative channel of the proteasomes. At this point, autophagy will become the main clearance route for these insoluble protein aggregates [3]. Autophagy is a bulk degradative system characterized by the engulfment of cargo molecules by autophagosomes, which are doublemembrane vesicular structures that have the capacity to engulf protein aggregates. After engulfment, the autophagosomes then fuse with cytoplasmic lysosomes containing acid hydrolases, and the content is subsequently degraded $[3,6]$. The general belief is that the UPS is responsible for the degradation of short-lived proteins and soluble misfolded proteins whereas autophagy eliminates long-lived proteins, insoluble protein aggregates and even whole organelles [6]. The two intracellular protein quality control / recycling systems were once thought to be independent of each other in components, action mechanisms, and substrate selectivity. However, recent advances in research have demonstrated that the UPS and autophagy actually functionally cooperate with each other in a single proteolytic network to maintain proteostasis [7-11]. Generally speaking, impairment to either the UPS or autophagy will lead to a compensatory upregulation of the other system [6]. This is at least partially due to common molecular players such as ATG16 and p62 in both systems which facilitate crosstalk and interplay between the two systems [7-11].

\section{Autophagy is Closely Associated with PolyQ diseases}

Autophagy was first identified as a self-consuming cellular process, which involves the degradation of proteins, lipids, and even entire organelles by lysosomal lytic enzymes. Autophagy is constitutively active at low levels, but it can be greatly induced by various forms of cellular stress, such as starvation, DNA damage, organelle damage and the aggregation of misfolded proteins [12]. Subsequently, autophagy was also found to be involved in the degradation of misfolded proteins as a cytoprotective response during neurodegeneration $[12,13]$. In particular, autophagy is closely associated with the degradation of mutant proteins and amelioration of cytotoxicity in polyQ diseases, including but limited to Huntington's disease and spinocerebellar ataxia 3 (a.k.a. Machado-Joseph disease) [14-18]. In a recent study, Rubinsztein and colleagues have demonstrated that the endogenous polyQ domain of the deubiquitinase ATXN3/ataxin-3 enables its interaction with BECN1/beclin 1, which is a major autophagy activator [19]. Thus, when this interaction is disrupted by mutant proteins with expanded polyQ tracts, it can result in decreased autophagy, likely by compromising autophagosome formation. This in turn may accelerate pathogenesis due to impairment of the clearance of aggregate-prone mutant polyQ proteins [19].

\section{PolyQ Protein Multimers and Microaggregates, but not Large Aggregates, may be the True Toxic Species in polyQ Diseases}

In another recent polyQ diseases study, Chan and colleagues discovered a bisamidine compound capable of deaggregating polyQ proteins and suppressing cell death. The compound was termed as Anti-polyQ-Aggregation for Machado-Joseph-Associated Neurodegeneration (AQAMAN) [20]. Interestingly, inhibition of autophagy abolished AQAMAN's effect on cell death suppression even though polyQ proteins were deaggregated by AQAMAN [20]. When autophagy was inhibited by the PI3K inhibitor, wortmannin, it should theoretically lead to 
a compensatory upregulation of the UPS, which may help with the clearance of at least the soluble polyQ protein monomers. However, Chan and colleagues found that, when autophagy was inhibited, AQAMAN lost its ability to rescue polyQ-induced cell death. This was not due to failure of deaggregation, as it has been shown by immunocytochemistry that AQAMAN was still able to reduced polyQ protein aggregation under the effect of wortmannin [20]. This indicated that some kind of soluble, invisible, deaggregated polyQ proteins remained in the cell and was causing cell death. This is consistent with another previous study, which suggested that the deaggregated polyQ proteins are still cytotoxic [21]. In fact, it was reported that large protein aggregates, at least in their early stages, may not be cytotoxic, but the smaller microaggregates may be the actual major toxic species $[22,23]$. These toxic protein species might be existing as polyQ protein multimers or microaggregates, and the UPS was unable to degrade them. Autophagy was necessary for the clearance of these toxic species from the cell.

\section{Autophagy: The True Hero that Eradicates "Unseen Dangers" for the Cell}

A classical view is that autophagy is mostly responsible for degrading the visible, insoluble protein aggregates, while the UPS degrades most of the soluble proteins. But in truth, perhaps the main source of cytotoxicity in protein aggregate-associated neurodegenerative diseases may actually come from the soluble protein multimers or semi-soluble microaggregates of mutant proteins. The UPS can probably degrade the protein monomers, which may help with preventing accumulation. However, the UPS is unable to degrade multimers and microaggregates, and thus the vital task of degrading these soluble/semisoluble toxic species depends solely on autophagy. Therefore, apart from degrading the visible protein aggregates (which may or may not actually be toxic), autophagy also acts like a hero in the dark that eradicates the true "unseen dangers" for the cell.

\section{References}

1. Pedersen JT, Heegaard NH (2013) Analysis of protein aggregation in neurodegenerative disease. Anal Chem 85(9): 4215-4227.

2. Chen ZS, Li L, Peng S, Chen FM, Zhang Q, et al. (2018) Planar cell polarity gene Fuz triggers apoptosis in neurodegenerative disease models. EMBO Rep 19(9): e4540.
3. Rubinsztein DC (2006) The roles of intracellular protein-degradation pathways in neurodegeneration. Nature 443(7113): 780-786.

4. Kleiger G, Mayor T (2014) Perilous journey: a tour of the ubiquitin-proteasome system. Trends Cell Biol 24(6): 352-359.

5. Dikic I (2017) Proteasomal and Autophagic Degradation Systems. Annu Rev Biochem 86: 193224.

6. Kocaturk NM, Gozuacik D (2018) Crosstalk between Mammalian Autophagy and the Ubiquitin-Proteasome System. Front Cell Dev Biol 6: 128.

7. Ji CH, Kwon YT (2017) Crosstalk and Interplay between the Ubiquitin-Proteasome System and Autophagy. Mol Cells 40(7): 441-449.

8. Nam T, Han JH, Devkota S, Lee HW (2017) Emerging Paradigm of Crosstalk between Autophagy and the Ubiquitin-Proteasome System. Mol Cells 40(12): 897905.

9. Blasiak J, Pawlowska E, Szczepanska J, Kaarniranta K (2019) Interplay between Autophagy and the Ubiquitin-Proteasome System and Its Role in the Pathogenesis of Age-Related Macular Degeneration. Int J Mol Sci 20(1).

10. Xiong Q, Li W, Li P, Yang M, Wu C, et al. (2018) The Role of ATG16 in Autophagy and The Ubiquitin Proteasome System. Cells 8(1).

11. Zientara-Rytter K, Subramani S (2019) The Roles of Ubiquitin-Binding Protein Shuttles in the Degradative Fate of Ubiquitinated Proteins in the UbiquitinProteasome System and Autophagy. Cells 8(1).

12. Rubinsztein DC, Gestwicki JE, Murphy LO, Klionsky DJ (2007) Potential therapeutic applications of autophagy. Nat Rev Drug Discov 6(4): 304-312.

13. Fujikake N, Shin M, Shimizu S (2018) Association between Autophagy and Neurodegenerative Diseases. Front Neurosci 12: 255.

14. Jimenez-Sanchez M, Thomson F, Zavodszky E, Rubinsztein DC (2012) Autophagy and polyglutamine diseases. Prog Neurobiol 97(2): 67-82.

15. Martin DD, Ladha S, Ehrnhoefer DE, Hayden MR (2015) Autophagy in Huntington disease and 
huntingtin in autophagy. Trends Neurosci 38(1): 2635.

16. Croce KR, Yamamoto A (2019) A role for autophagy in Huntington's disease. Neurobiol Dis 122: 16-22.

17. Bilen J, Bonini NM (2007) Genome-wide screen for modifiers of ataxin-3 neurodegeneration in Drosophila. PLoS Genet 3(10): 1950-1964.

18. Ou Z, Luo M, Niu X, Chen Y, Xie Y, et al. (2016) Autophagy Promoted the Degradation of Mutant ATXN3 in Neurally Differentiated Spinocerebellar Ataxia-3 Human Induced Pluripotent Stem Cells. Biomed Res Int 2016: 6701793.

19. Ashkenazi A, Bento CF, Ricketts T, Vicinanza M, Siddiqi F, et al. (2017) Polyglutamine tracts regulate beclin 1-dependent autophagy. Nature 545(7652): 108-111.
20. Hong H, Koon AC, Chen ZS, Wei Y, An Y, et al. (2019) AQAMAN, a bisamidine-based inhibitor of toxic protein inclusions in neurons, ameliorates cytotoxicity in polyglutamine disease models. J Biol Chem 294(8): 2757-2770.

21. Hands SL, Wyttenbach A (2010) Neurotoxic protein oligomerisation associated with polyglutamine diseases. Acta Neuropathol 120(4): 419-437.

22. Arrasate M, Mitra S, Schweitzer ES, Segal MR, Finkbeiner S (2004) Inclusion body formation reduces levels of mutant huntingtin and the risk of neuronal death. Nature 431(7010): 805-810.

23. Tanaka M, Kim YM, Lee G, Junn E, Iwatsubo T, et al. (2004) Aggresomes formed by alpha-synuclein and synphilin-1 are cytoprotective. J Biol Chem 279(6): 4625-4631. 